\title{
New generation multi-modal antidepressants: focus on vortioxetine for major depressive disorder
}

This article was published in the following Dove Press journal:

Neuropsychiatric Disease and Treatment

19 February 2014

Number of times this article has been viewed

\author{
Cornelius L Katona' \\ Cara P Katona ${ }^{2}$ \\ 'Division of Psychiatry, University \\ College London, ${ }^{2}$ North Central \\ London Psychiatry Training \\ Programme, London, UK
}

Correspondence: Cornelius L Katona UCL Division of Psychiatry, Charles Bell House, 67-73 Riding House Street, London WIW 7EJ, UK

Fax +44 I3048I25I4

Email c.katona@ucl.ac.uk

\begin{abstract}
Vortioxetine is a novel antidepressant with effects on multiple 5-HT receptors and on the serotonin transporter. This paper reviews preclinical and clinical evidence regarding its mechanism of action, its tolerability, and its efficacy in treating major depression. Clinical studies indicate that vortioxetine is effective in the treatment of major depression, though there is no suggestion of superiority over active comparators. There may be a clinically meaningful advantage in terms of tolerability.
\end{abstract}

Keywords: vortioxetine, major depression, review

\section{Overview of current treatment strategies for major depressive disorder (MDD) and their limitations}

MDD is one of the leading causes of disability in both the developed and the developing world. It has consistently been found to be associated with significant reductions in quality of life, impaired work productivity, reduced social functioning, poor physical health, and substantial direct and indirect economic costs. According to the World Health Organization's Global Burden of Disease project, ${ }^{1}$ MDD will become the second leading cause of disability worldwide within the next 10 years.

Antidepressants, together with evidence-based psychological therapies such as cognitive behavioral therapy, are important components of current treatment for major depression. Their efficacy is, however, quite limited. Only about $30 \%-40 \%$ achieve a full remission after a single adequate course of antidepressants. A further third have a clinically significant response to antidepressant therapy but have residual symptoms which limit their social functioning and increase their risk of relapse. ${ }^{2}$

Treatment adherence is a further problem. ${ }^{3}$ Fewer than half of patients with MDD take their antidepressants consistently and for the full recommended duration. This reflects the delayed onset of action associated with antidepressant use and their considerable side effect burden. Common side effects (which are often prominent in the weeks before any clinical response becomes evident) include weight gain, sexual dysfunction, nausea, headache, and sleep disturbances. There is therefore, a clear need for novel antidepressants with distinct mechanisms of action and improved side effect profiles.

Vortioxetine, an antidepressant produced and co-marketed by Lundbeck and Takeda, was approved by the US Food and Drug Administration under the trade name Brintellix. At the time of writing, vortioxetine remains under consideration by the European licensing authorities. This paper reviews the mechanism of action 
of vortioxetine, the evidence regarding its safety and efficacy, and its potential place in the depression treatment armamentarium.

\section{Novel mechanism of action of vortioxetine}

Vortioxetine was developed as one of a series of compounds developed from halogenated benzenes and was intended to have combined effects on multiple 5-HT receptors and on the serotonin transporter. It has been shown in recombinant cell lines to combine 5- $\mathrm{HT}_{3}$ and 5- $\mathrm{HT}_{7}$ receptor antagonism, $5-\mathrm{HT}_{1 \mathrm{~B}}$ receptor partial agonism, 5- $\mathrm{HT}_{1 \mathrm{~A}}$ receptor agonism, and serotonin transporter inhibition. ${ }^{4}$

\section{Review of pharmacology, mode of action, and pharmacokinetics of vortioxetine}

Mørk et $\mathrm{al}^{5}$ assessed the effects of vortioxetine on brain neurotransmitter levels in vivo in freely-moving rat models predictive of antidepressant and anxiolytic-like activity. They confirmed that vortioxetine had multimodal serotonergic actions including partial 5- $\mathrm{HT} 1_{\mathrm{B}}$ receptor agonism, $5-\mathrm{HT}_{7}$ antagonism, 5- $\mathrm{HT}_{3}$ antagonism, and inhibition of the serotonin transporter.

Bétry et $\mathrm{al}^{6}$ used electrophysiological and autoradiography studies in male Sprague-Dawley rats to examine the acute and chronic effects of vortioxetine on 5-HT neuronal firing activity and to compare them with the effects of the selective serotonin reuptake inhibitor fluoxetine. Vortioxetine caused a rapid decrease in spontaneous firing of 5-HT neurons in the dorsal raphe nucleus, which (in contrast with the effects of fluoxetine) recovered within 1 day. Subsequent autoradiographic studies indicated that 5-HT neuronal firing was inhibited by vortioxetine at doses that only partially blocked the serotonin transporter. Vortioxetine administered for 3 days desensitized 5- $\mathrm{HT}_{1 \mathrm{~A}}$ autoreceptors to the effects of the $5-\mathrm{HT}_{1 \mathrm{~A}}$ agonist flexinoxan. The authors concluded that vortioxetine inhibited 5-HT neuronal activity indirectly by inducing the release of extracellular 5-HT and suggested that $5-\mathrm{HT}_{3}$ receptor antagonism was also an important aspect of its mechanism of action.

Pehrson et $\mathrm{al}^{7}$ examined the effects of acute and subchronic treatment with vortioxetine (compared with escitalopram) on extracellular 5-HT norepinephrine (NE) and dopamine (DA) levels in a rat ventral hippocampus (vHC), medial prefrontal cortex, and nucleus accumbens, as well as its effects on NE and DA neuronal firing in the locus coeruleus and in the ventral tegmental area. 5-HT levels were increased by vortioxetine (most markedly in the vHC) despite quite low 5-HT receptor occupancy. NE and DA levels were also somewhat increased in the $\mathrm{vHC}$ and medial prefrontal cortex but not in the nucleus accumbens. The authors concluded that vortioxetine had two main mechanisms of action (5-HT receptor modulation and serotonin transporter inhibition) through which it induced region-dependent increases in the concentration of multiple neurotransmitters.

Single dose human studies show that vortioxetine is extensively metabolized in the liver, and is a substrate for a range of cytochrome P450 (CYP450) isoforms. ${ }^{8}$ In vitro studies using human liver cells indicate that vortioxetine has no CYP450 inducing or inhibitory effects. ${ }^{9,10}$

Areberg et $\mathrm{al}^{11}$ carried out an extensive human pharmacokinetic study. They administered vortioxetine by mouth and intravenously in a total of 97 healthy volunteers aged 18-51 years (median 24). Vortioxetine had an extended absorption phase and medium clearance. The volume of distribution was large, indicating that vortioxetine is lipophilic with high affinity for peripheral tissues. Once a steady state was reached, plasma vortioxetine levels varied little throughout the day. The absolute bioavailability was $75 \%$. Mean elimination half-life following oral administration was 57 hours. In single-dose studies, women had higher exposure to vortioxetine (as measured both by peak concentration and area under the curve), but the differences were small after correcting for weight. The gender difference was not statistically significant in multiple-dose studies. The authors also noted that previous studies had reported that food had no effect on the pharmacokinetics of vortioxetine.

Chen et $\mathrm{al}^{12}$ carried out multiple studies in healthy human volunteers to evaluate potential pharmacokinetic interactions between vortioxetine and coadministered agents with a range of activity as inhibitors, inducers, or substrates for CYP450 subtypes. They identified potentially significant interactions in the form of increased vortioxetine levels when it was coadministered with bupropion (CYP2D6 inhibitor and CYP2B6 substrate), fluconazole (inhibitor of CYP450 2C9, 2C19, and 3A), and ketoconazole (CYP3A and $\mathrm{P}$-glycoprotein inhibitor) and decreased vortioxetine levels when it was coadministered with rifampicin (CYP inducer). The authors considered that only the interactions with bupropion and rifampicin were likely to be sufficiently significant to warrant possible dosage adjustment. The US datasheet (http://www.accessdata.fda.gov/drugsatfda docs/ label/2013/204447s0001bl.pdf) recommends reducing the dose of vortioxetine when giving it in combination with powerful CYP2D6 inhibitors such as bupropion, fluoxetine, 
paroxetine, and quinidine, and increasing it when it is administered in combination with powerful CYP inducers such as carbamazepine and phenytoin.

\section{Efficacy studies, including any comparative studies Animal studies}

Animal studies suggest that not only does vortioxetine have an antidepressant-like profile but also that - unlike many established antidepressants - it may have memoryenhancing effects.

The putative antidepressant effects of vortioxetine were confirmed in a behavioral model of depression ${ }^{13}$ in which vortioxetine (as well as amitriptyline) significantly reduced immobility in rats in the forced swim test model of induced depression, whereas neither fluoxetine nor duloxetine did so. In keeping with this, Guilloux et $\mathrm{al}^{14}$ assessed the effects of vortioxetine in three mouse models of anxiety and depression-like behavior - the forced swim test, the open field test, and the novelty-suppressed feeding paradigm. Both acute and repeated dosing with vortioxetine produced antidepressant and anxiolytic effects greater than those produced by fluoxetine and comparable (in the open field test) with those produced by diazepam. Vortioxetine also increased cell proliferation and cell survival of immature granule cells in the dentate gyrus of the hippocampus and stimulated their maturation. These effects were observed despite low levels of serotonin receptor occupancy.

Mørk et al ${ }^{15}$ examined the effect of vortioxetine on memory in rats using contextual fear conditioning and novel object recognition tests. They found that vortioxetine enhanced both contextual and episodic memory and that these effects were unrelated to pain sensitivity. They also found that vortioxetine increased extracellular levels of acetylcholine and histamine. They concluded that these effects were consistent with the multiple neurotransmitter effects induced by vortioxetine via $5-\mathrm{HT}_{3}$ and $5-\mathrm{HT}_{7}$ receptor antagonism and 5-HT $1 \mathrm{~A}$ receptor agonism. In keeping with this, du Jardin et al ${ }^{16}$ found that vortioxetine dose-dependently reversed memory deficits in female Long-Evans rats (as measured by object recognition and Y-maze spontaneous alternation tests) induced by 5 -HT depletion.

\section{Clinical studies}

Vortioxetine has been extensively evaluated as a potential treatment for major depression. We have identified five placebo-controlled short-term (6-8 weeks) studies of vortioxetine in younger adult patients with major depression.
Of these, three also had an active comparator (duloxetine or venlafaxine). In addition there has been an acute placebocontrolled study in the elderly and two longer-term relapse prevention studies (one of them randomized and placebocontrolled). Vortioxetine has also been subjected to randomized controlled comparison with agomelatine.

Baldwin et a ${ }^{17}$ compared vortioxetine $(2.5 \mathrm{mg}, 5 \mathrm{mg}$, or $10 \mathrm{mg}$ ) with placebo (with duloxetine $60 \mathrm{mg}$ as an active comparator) in 766 patients with major depression and a Montgomery-Åsberg Depression Rating Scale (MADRS) score of at least 26. On the predefined (last observation carried forward) primary analysis, the differences between vortioxetine (or duloxetine) and placebo were not significant. In mixed model repeat measures analyses however, the two higher doses of vortioxetine were significantly superior to placebo, as was duloxetine.

Mahableshwarkar et $\mathrm{al}^{18}$ evaluated the safety and efficacy of vortioxetine $(2.5 \mathrm{mg}$ or $5 \mathrm{mg}$ ) against placebo and against the active comparator duloxetine $(60 \mathrm{mg})$ in an 8 -week study in a total of 611 participants. The primary outcome variable was the change from baseline in the 24-item Hamilton Depression Scale (HAMD-24). For vortioxetine, although there was substantial reduction in the HAMD-24 scores, the difference against placebo was not statistically significant for either dose. In contrast, duloxetine showed superiority from placebo at 8 weeks (and also at 6 weeks). Vortioxetine was well-tolerated with low dropout and side-effect rates.

Jain et $\mathrm{al}^{19}$ randomized 600 people to $5 \mathrm{mg}$ vortioxetine or placebo in a 6-week study. Participants were required to have a MADRS score of at least 30 and a diagnosis of a major depressive episode. The primary endpoints were change from baseline in HAMD-24 total score at 6 weeks and sequential change week by week in HAMD-24 score. Four hundred eighty $(80 \%)$ participants completed the study. Withdrawal rates (both for adverse events and for lack of efficacy) were similar for vortioxetine and for placebo. There were no significant differences between vortioxetine and placebo in either of the primary outcome variables, though post hoc analyses revealed greater improvement on vortioxetine in the subgroup with high baseline anxiety scores.

Alvarez et a ${ }^{20}{ }^{20}$ evaluated vortioxetine $(5 \mathrm{mg}$ or $10 \mathrm{mg}$ fixed dose) in a 6-week comparison against placebo, with venlafaxine (225 $\mathrm{mg}$ slow release) as an active comparator. The primary outcome variable was mean change in MADRS total score. Both doses of vortioxetine were significantly superior to placebo at the 6-week endpoint, with mean MADRS differences from placebo of 5.9 for $5 \mathrm{mg}$ and 5.7 for $10 \mathrm{mg}$. Venlafaxine was also superior to placebo (mean difference in 
6-week MADRS score of 6.4). Secondary outcome measures (response rates as measured by HAMD-24 and MADRS) also showed superiority for both doses of vortioxetine (and for venlafaxine) against placebo.

The efficacy of vortioxetine (at doses of $1 \mathrm{mg}, 5 \mathrm{mg}$, and $10 \mathrm{mg}$ a day) against placebo was evaluated by Henigsberg et $\mathrm{al}^{21}$ in an 8-week trial involving a total of 560 participants. Participants fulfilled Diagnostic and Statistical Manual of Mental Disorders Fourth Edition Text Revision criteria for major depressive episode and had a minimum MADRS score of 26 . The highest dose of vortioxetine (10 mg) was significantly superior to placebo on the primary endpoint measure, reduction from baseline in the HAMD-24 total score at week $8(P<0.001)$. There were also greater improvements with all doses of vortioxetine than with placebo for most depression-related variables.

In a 12-week flexible dose study ${ }^{22}$ in 493 patients with MDD who had failed to respond adequately to a selective serotonin reuptake inhibitor or a serotonin-norepinephrine reuptake inhibitor (SNRI), vortioxetine (10-20 mg) was found to be superior to agomelatine (25-50 mg). Change in MADRS total score at 8 weeks was the primary outcome measure. Vortioxetine also showed superiority in several secondary outcome measures.

In a three-arm comparison with duloxetine $(60 \mathrm{mg})$ and placebo over 8 weeks in patients aged 65 and over, vortioxetine ( $5 \mathrm{mg}$ fixed dose) showed significantly greater improvement than placebo on the predefined primary efficacy endpoint (ie, analysis of covariance, last observation carried forward) of HAMD-24 score at week $8 .^{23}$ Duloxetine was also superior to placebo at week 8 . Vortioxetine also showed superiority to placebo in a range of cognitive tests (processing speed, verbal learning, and memory), in keeping with the findings from animal studies.

It is perhaps noteworthy that in three of the above studies, ${ }^{18,20,23}$ although formal statistical comparison between active drugs was not reported, there was a trend for the SNRI active comparator to be associated with numerically superior outcomes to vortioxetine.

In common with other antidepressants, vortioxetine appears to be effective in preventing depressive relapse. Baldwin et $\mathrm{al}^{24}$ reported on a 52-week, open-label extension study which followed an 8-week lead-in. The MADRS total score was used as the primary outcome variable. In total, $328 / 535$ patients $(61.3 \%)$ completed the study, representing a total of 393 patient years of exposure to vortioxetine. At the point of entry to the extension study, participants had a mean MADRS total score of 13.5 \pm 8.7 . During the subsequent year, the mean MADRS total score in completers decreased by approximately 8 points to $5.5 \pm 6.0$ at week 52 (OC) and the proportion of responders rose from $63 \%$ to $94 \%$. Remission rate in completers ( $42 \%$ at the start of the study) had increased to $83 \%$ (OC). Relapse rate in those in remission at the start of the study $(n=226)$ was $9.7 \%$.

Boulenger et $\mathrm{al}^{25}$ confirmed that vortioxetine was effective in preventing depressive relapse in a study of 396 patients who, after achieving remission during 12-weeks of open-label treatment with 5-10 $\mathrm{mg}$ vortioxetine, were randomized to either placebo or vortioxetine (fixed dose of $5 \mathrm{mg}$ or $10 \mathrm{mg}$ as determined during the open-label phase). The primary efficacy variable was time to relapse (defined on the basis of a MADRS score of $>21$ or clinically judged lack of efficacy). Overall, fewer patients (13\% versus $26 \%$ ) relapsed on vortioxetine than on placebo $(P=0.013)$. Nausea was the only side effect that occurred significantly more often with vortioxetine than with placebo during the double-blind phase of the study.

\section{Safety and tolerability}

Clinical studies suggest that vortioxetine has a good safety and tolerability profile. Henigsberg et $\mathrm{al}^{21}$ reported that vortioxetine was generally well-tolerated; the most common adverse events associated with it were nausea, headache, and dizziness. Alvarez et $\mathrm{al}^{20}$ noted higher adverse-event-related withdrawal rates on venlafaxine (14\%) than for either dose of vortioxetine $(3 \%$ on $5 \mathrm{mg}$ and $7 \%$ on $10 \mathrm{mg}$ ) or for placebo (4\%). Sexual dysfunction on vortioxetine was at a similar rate to that found on placebo. In keeping with this, Baldwin et $\mathrm{al}^{17}$ reported sexual dysfunction was present in very few participants $(2 \%-4 \%)$ at all three vortioxetine doses they studied, compared with $14 \%$ of those on duloxetine. In their comparison with agomelatine, Häggström et $\mathrm{al}^{22}$ found that vortioxetine was better tolerated overall (adverse-event-related withdrawal dates of 5.9\% versus $9.5 \%$ ). Vortioxetine is also well-tolerated in older people. Katona et $\mathrm{a}^{23}$ found that the adverse-event-related withdrawal rates were $5.8 \%$ for vortioxetine compared with $2.8 \%$ (placebo) and $9.9 \%$ (duloxetine). Nausea was the only adverse event with a significantly higher incidence on vortioxetine $(21.8 \%)$ than placebo $(8.3 \%)$. In contrast, the incidence of nausea, constipation, dry mouth, hyperhidrosis, and somnolence were all higher for duloxetine than placebo.

Vortioxetine appears not to affect driving performance. Theunissen et $\mathrm{a}^{26}$ examined its effects (at a dose of $10 \mathrm{mg} /$ day) on driving, cognitive, and psychomotor performance (in a randomized controlled comparison with mirtazapine $30 \mathrm{mg}$ /day and placebo) in 24 healthy subjects over a 15-day period. Vortioxetine did not cause any impairment in any of 
the measures at either of the time points (day 2 and day $16-$ ie, the morning after the last dose). In contrast, mirtazapine (which is of course relatively sedating) was associated with cognitive and psychomotor impairment on day 2 , but this was no longer apparent on day 16.

\section{Conclusion and place in therapy}

Vortioxetine represents a new class of antidepressant. It has multiple actions that enhance serotonergic activity. This appears to have "knock-on" effects on other neurotransmitters implicated in the causation and maintenance of depressive disorders. Vortioxetine is well-tolerated and appears to have relatively little potential for adverse drug interactions. Clinical studies indicate that it is effective in the treatment of major depression. Though there is no suggestion of superiority over active comparators (and some suggestion that SNRIs may show greater efficacy), most studies suggest that there is a clinically meaningful advantage in terms of tolerability. Incidence of sexual side-effects appears particularly low. Older people tolerate vortioxetine well, and there is some evidence that it may have cognitive benefits.

\section{Disclosure}

Cornelius L Katona is currently involved in a clinical trial of vortioxetine and has given paid talks and received payments for consultancy work by Lundbeck. Cara P Katona has no potential conflicts of interest.

\section{References}

1. World Health Organization. The Global Burden of Disease (2004 update). Geneva: World Health Organization; 2008. Available from: http://www. who.int/healthinfo/global_burden_disease/GBD_report_2004update_ full.pdf. Accessed November 20, 2013.

2. Rozensweig-Lipson S, Beyer CE, Hughes ZA, et al. Differentiating antidepressants of the future: efficacy and safety. Pharmacol Ther. 2007;113(1):134-143.

3. Papakostas GI. Limitations of contemporary antidepressants: tolerability. J Clin Psychiatry. 2007;68 Suppl 10:11-17.

4. Bang-Andersen B, Ruhland T, Jørgensen M, et al. Discovery of 1-[2(2,4-dimethylphenylsulfanyl)phenyl]piperazine (Lu AA21004): a novel multimodal compound for the treatment of major depressive disorder. J Med Chem. 2011;54(9):3206-3221.

5. Mørk A, Pehrson L, Brennum LT, et al. Pharmacological effects of LU AA21004: a novel multimodal compound for the treatment of major depressive disorder. J Pharmacol Exp Ther. 2012;340(3):666-675.

6. Bétry C, Pehrson Al, Etiévant A, Ebert B, Sánchez C, Hadderji N. The rapid recovery of 5-HT cell firing induced by the antidepressant vortioxetine involves 5- $\mathrm{HT}_{3}$ receptor antagonism Int J Neuropsychopharmacol. 2013;16(5):1115-1127.

7. Pehrson AL, Cremers T, Bétry C, et al. Lu AA21004, a novel multimodal antidepressant, produces regionally selective increases of multiple neurotransmitter - a rat microdialysis and electrophysiology study. Eur Neuropsychopharmacol. 2013;23(2):133-145.

8. Hvenegaard MG, Bang-Andersen B, Pedersen H, Jørgensen M, Püschl A, Dalgaard L. Identification of the cytochrome P450 and other enzymes involed in the in vitro metabolism of a novel antidepressant, Lu AA21004. Drug Metab Dispos. 2012;40(7):1357-1365.
9. Wang Y, Munsaka M, Hanson E, et al. The effect of multiple-doses of fluconazole, or ketoconazole, on the single dose pharmacokinetic profile in healthy adult subjects (abstract). AAPS J. 2010;12(S2):T2381.

10. Wang Y, Wojtkowski T, Ross G, et al. Effect of multiple doses of rifampicin on the single dose pharmacokinetics of Lu AA21004 [abstract]. J Clin Pharmacol. 2010;50:1082.

11. Areberg J, Søgaard B, Højer AM. The clinical pharmacokinetics of Lu AA21004 and its major metabolite in healthy young volunteers. Basic Clin Pharmacol Toxicol. 2012;111(3):198-205.

12. Chen G, Lee R, Højer AM, Buchbjerg JK, Serenko M, Zhao Z. Pharmacokinetic drug interactions involving vortioxetine ( $\mathrm{Lu}$ AA21004), a multimodal antidepressant Clin Drug Investig. 2013; 33(10):727-736.

13. Li Y, Kaaby KF, Sánchez C, Gulinello M. Serotonergic receptor mechanisms underlying antidepressant-like action in the progesterone withdrawal model of hormonally induced depression in rats. Behav Brain Res. 2013;256:520-528.

14. Guilloux JP, Mendez-David I, Pehrson A, et al. Antidepressant and anxiolytic potential of the multimodal antidepressant vortioxetine ( $\mathrm{Lu}$ AA21004) assessed by behavioural and neurogenesis outcomes in mice. Neuropharmacology. 2013;73:147-159.

15. Mørk A, Montezinho LP, Miller S, et al. Vortioxetine (LU 21004), a novel multimodal antidepressant, enhances memory in rats. Pharmacol Biochem Behav. 2013;105:41-50.

16. du Jardin KG, Jensen JB, Sanchez C, Pehrson AL. Vortioxetine dosedependently reverses 5-HT depletion-induced deficits in spatial working and object recognition memory: A potential role for 5-HT1A receptor agonism and 5-HT3 receptor antagonism. Eur Neuropsychopharmacol. Epub August 2, 2013.

17. Baldwin DS, Loft H, Dragheim M. A randomised, double-blind, placebo controlled, duloxetine-referenced, fixed-dose study of three dosages of Lu AA21004 in acute treatment of major depressive disorder (MDD). Eur Neuropsychopharmacol. 2012;22(7):482-491.

18. Mahableshwarkar AR, Jacobsen PL, Chen Y. A randomized, doubleblind trial of $2.5 \mathrm{mg}$ and $5 \mathrm{mg}$ vortioxetine (Lu AA21004) versus placebo for 8 weeks in adults with major depressive disorder. Curr Med Res Opin. 2013;29(3):217-226.

19. Jain R, Mahableshwarkar AR, Jacobsen PL, Chen Y, Thase ME. A randomized, double-blind, placebo-controlled 6-wk trial of the efficacy and tolerability of $5 \mathrm{mg}$ vortioxetine in adults with major depressive disorder. Int J Neuropsychopharmacol. 2013;16(2):313-321.

20. Alvarez E, Perez V, Dragheim M, Loft H, Artigas F. A double-blind, randomized, placebo-controlled, active reference study of Lu AA21004 in patients with major depressive disorder. Int J Neuropsychopharmacol. 2012;15(5):589-600.

21. Henigsberg N, Mahableshwarkar AR, Jacobsen P, Chen Y, Thase ME. A randomized, double-blind, placebo-controlled 8-week trial of the efficacy and tolerability of multiple doses of Lu AA21004 in adults with major depressive disorder. J Clin Psychiatry. 2012;73(7):953-959.

22. Häggström L, Nielsen RZ, Dragheim M. Randomized double-blind study of vortioxetine versus agomelatine in adults with MDD after inadequate response to SSRI or SNRI treatment. European Psychiatry. 2013;28(Supp 1):1.

23. Katona C, Hansen T, Olsen CK. A randomized, double-blind, placebocontrolled, duloxetine-referenced, fixed-dose study comparing the efficacy and safety of Lu AA21004 in elderly patients with major depressive disorder. Int Clin Psychopharmacol. 2012;27(4):215-223.

24. Baldwin DS, Hansen T, Florea I. Vortioxetine (Lu AA21004) in the long-term open-label treatment of major depressive disorder. Curr Med Res Opin. 2012;28(10):1717-1724.

25. Boulenger J-P, Loft H, Florea A. A randomized clinical study of Lu AA21004 in the prevention of relapse in patients with major depressive disorder. J Psychopharmacol. 2012;26(11):1408-1416.

26. Theunissen EL, Street D, Højer AM, Vermeeren A, van Oers A, Ramaekers JG. A randomized trial on the acute and steady-state effects of a new antidepressant, vortioxetine (Lu AA21004), on actual driving and cognition. Clin Pharmacol Ther. 2013;93(6):493-501. 


\section{Publish your work in this journal}

Neuropsychiatric Disease and Treatment is an international, peerreviewed journal of clinical therapeutics and pharmacology focusing on concise rapid reporting of clinical or pre-clinical studies on a range of neuropsychiatric and neurological disorders. This journal is indexed on PubMed Central, the 'PsycINFO' database and CAS.

The manuscript management system is completely online and includes a very quick and fair peer-review system, which is all easy to use. Visit http://www.dovepress.com/testimonials.php to read real quotes from published authors.

\footnotetext{
Submit your manuscript here: http://www.dovepress.com/neuropsychiatric-disease-and-treatment-journal
} 